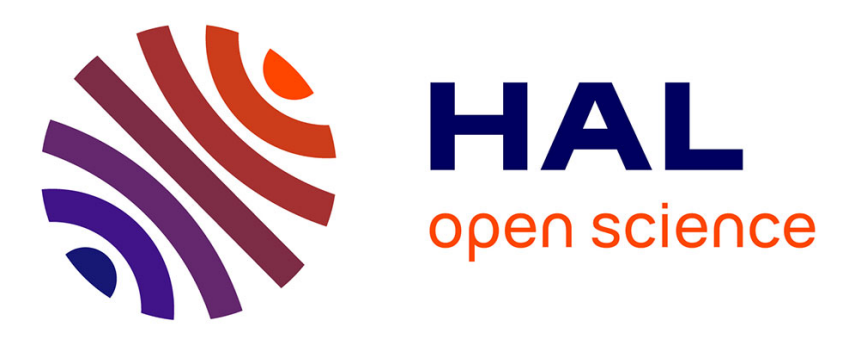

\title{
Data redistribution algorithms for heterogeneous processor rings
}

Hélène Renard, Yves Robert, Frédéric Vivien

\section{To cite this version:}

Hélène Renard, Yves Robert, Frédéric Vivien. Data redistribution algorithms for heterogeneous processor rings. International Journal of High Performance Computing Applications, 2006, 20 (1), pp.31-43. 10.1177/1094342006061887 . hal-00804395

\section{HAL Id: hal-00804395 \\ https://hal.inria.fr/hal-00804395}

Submitted on 13 Nov 2018

HAL is a multi-disciplinary open access archive for the deposit and dissemination of scientific research documents, whether they are published or not. The documents may come from teaching and research institutions in France or abroad, or from public or private research centers.
L'archive ouverte pluridisciplinaire HAL, est destinée au dépôt et à la diffusion de documents scientifiques de niveau recherche, publiés ou non, émanant des établissements d'enseignement et de recherche français ou étrangers, des laboratoires publics ou privés. 


\title{
Data redistribution algorithms for heterogeneous processor rings
}

\author{
Hélène Renard Yves Robert Frédéric Vivien \\ LIP, UMR CNRS-INRIA-UCBL 5668, ENS Lyon, France \\ email: \{Helene.Renard | Yves.Robert | Frederic.Vivien\}@ens-lyon.fr
}

\begin{abstract}
We consider the problem of redistributing data on homogeneous and heterogeneous ring of processors. The problem arises in several applications, after each invocation of a load-balancing mechanism (but we do not discuss the loadbalancing mechanism itself). We provide algorithms that aim at optimizing the data redistribution, both for uni-directional and bidirectional rings. One major contribution of the paper is that we are able to prove the optimality of the proposed algorithms in all cases except that of a bi-directional heterogeneous ring, for which the problem remains open.
\end{abstract}

\section{Introduction}

In this paper, we consider the problem of redistributing data on a heterogeneous ring of processors. The problem typically arises when a load balancing phase must be initiated. Because either of variations in the resource performances (CPU speed, communication bandwidth) or in the system/application requirements (completed tasks, new tasks, migrated tasks, etc.), data must be redistributed between participating processors so that the current (estimated) load is better balanced. We do not discuss the loadbalancing mechanism itself (we take it as external, be it a system, an algorithm, an oracle, or whatever). Rather we aim at optimizing the data redistribution induced by the load- balancing mechanism.

We adopt the following abstract view of the problem. There are $n$ participating processors $P_{1}, P_{2}, \ldots, P_{n}$. Each processor $P_{k}$ initially holds $L_{k}$ atomic data items. The load-balancing system/algorithm/oracle has decided that the new load of $P_{k}$ should be $L_{k}-\delta_{k}$. If $\delta_{k}>0$, this means that $P_{k}$ now is overloaded and should send $\delta_{k}$ data items to other processors; if $\delta_{k}<0, P_{k}$ is under-loaded and should receive $-\delta_{k}$ data items from other processors. Of course there is a conservation law: $\sum_{k=1}^{n} \delta_{k}=0$. The goal is to determine the required communications and to organize them (what we call the data redistribution) in minimal time.

We assume that the participating processors are arranged along a ring, either unidirectional or bidirectional, and either with homogeneous or heterogeneous link bandwidths, hence a total of four different frameworks to deal with. There are two main contexts in which processor rings are useful. The first context is that of many applications which operate on ordered data, and where the order needs to be preserved. Think of a large matrix whose columns are distributed among the processors, but with the condition that each processor operates on a slice of consecutive columns. An overloaded processor $P_{i}$ can send its first columns to the processor $P_{j}$ that is assigned the slice preceding its own slice (and $P_{j}$ would append these columns to the end of its slice); similarly, $P_{i}$ can send its last columns to the processor which is assigned the next slice; ob- 
viously, these are the only possibilities. In other words, the ordered uni-dimensional data distribution calls for a uni-dimensional arrangement of the processors, i.e., along a ring.

The second context that may call for a ring is the simplicity of the programming. Using a ring, either uni- or bi-directional, allows for a simpler management of the data to be redistributed. Data intervals can be maintained and updated to characterize each processor load. Finally, we observe that parallel machines with a rich but fixed interconnection topology (hypercubes, fat trees, grids, to quote a few) are on the decline. Heterogeneous cluster architectures, which we target in this paper, have a largely unknown interconnection graph, with includes gateways, backbones, and switches, and modeling the communication graph as a ring is a reasonable, if conservative, choice.

As stated above, we discuss four cases for the redistribution algorithms. We delay the formal statement of the redistribution problems until Section 2, but we summarize the main results as follows. In the simplest case, that of a unidirectional homogeneous ring, we derive an optimal algorithm, and we prove its correctness in full details. Because the target architecture is quite simple, we are able to provide explicit (analytical) formulas for the number of data sent/received by each processor. The same holds true for the case of a bidirectional homogeneous ring, but the algorithm becomes more complicated. When assuming heterogeneous communication links, we still derive an optimal algorithm for the unidirectional case, but we have to use an asynchronous formulation. However, we are only able to solve the bidirectional case in the special case of light redistributions. We point out that one major contribution of the paper is the design of optimal algorithms, together with their formal proof of correctness: to the best of our knowledge, this is the first time that optimal algorithms are introduced.

The rest of the paper is organized as follows. In Section 2 we formally state the optimization problem. For homogeneous networks (all links have same capacity), the optimal algorithms are described in Section 3 (unidirectional ring) and in Section 5 (bidirectional ring). For heterogeneous networks, the optimal asynchronous unidirectional algorithm is presented in Section 4, and the linear-programming based optimal algorithm for light redistributions on bidirectional links is explained in Section 6. Section 7 is devoted to a survey of related work. In Section 8, we overview some simulation results that confirm the usefulness of data redistributions. Finally, Section 9 concludes the paper and highlights future work directions.

Due to page limits, we were not able to include all the proofs in this paper. The missing ones can be found in [30].

\section{Framework}

We consider a set of $n$ processors $P_{1}, P_{2}, \ldots, P_{n}$ arranged along a ring. The successor of $P_{i}$ in the ring is $P_{i+1}$, and its predecessor is $P_{i-1}$, where all indices are taken modulo $n$. For $1 \leq k, l \leq n$, $C_{k, l}$ denotes the slice of consecutive processors $C_{k, l}=P_{k}, P_{k+1}, \ldots, P_{l-1}, P_{l}$.

We denote by $c_{i, i+1}$ the capacity of the communication link from $P_{i}$ to $P_{i+1}$. In other words, it takes $c_{i, i+1}$ time-units to send an atomic data item from processor $P_{i}$ to processor $P_{i+1}$. In the case of a bidirectional ring, $c_{i, i-1}$ is the capacity of the link from $P_{i}$ to $P_{i-1}$. We use the oneport model for communications: at any given time, there are at most two communications involving a given processor, one sent and the other received. A given processor can simultaneously send and receive data, so there is no restriction in the unidirectional case; however, in the bidirectional case, a given processor cannot simultaneously send data to its successor and its predecessor; neither can it receive data from both sides. This is the only restriction induced by the model: any pair of communications that does not violate the one-port constraint can take place in parallel. 
Each processor $P_{k}$ initially holds $L_{k}$ atomic data items. After redistribution, $P_{k}$ will hold $L_{k}-\delta_{k}$ atomic data items. We call $\delta_{k}$ the unbalance of $P_{k}$. We denote by $\delta_{k, l}$ the total unbalance of the processor slice $C_{k, l}: \delta_{k, l}=$ $\delta_{k}+\delta_{k+1}+\cdots+\delta_{l-1}+\delta_{l}$.

Because of the conservation law of atomic data items, $\sum_{k=1}^{n} \delta_{k}=0$. Obviously the unbalance cannot be larger than the initial load: $L_{k} \geq \delta_{k}$. In fact, we suppose that any processor holds at least one data item, both initially $\left(L_{k} \geq 1\right)$ and after the redistribution $\left(L_{k} \geq 1+\delta_{k}\right)$ : otherwise we would have to build a new ring from the subset of resources still involved in the computation.

\section{Homogeneous unidirectional ring}

In this section, we consider a homogeneous unidirectional ring. Any processor $P_{i}$ can only send data items to its successor $P_{i+1}$, and $c_{i, i+1}=c$ for all $i \in[1, n]$. We first derive a lower bound on the running time of any redistribution algorithm. Then, we present an algorithm achieving this bound (hence optimal), and we prove its correctness.

\subsection{Lower bound}

We have the following bound on the optimal redistribution time:

Lemma 1. Let $\tau$ be the optimal redistribution time. Then:

$$
\tau \geq\left(\max _{\substack{1 \leq k \leq n, 0 \leq l \leq n-1}}\left|\delta_{k, k+l}\right|\right) \times c .
$$

Proof. The processor slice $C_{k, k+l}=P_{k}, P_{k+1}$, $\ldots, P_{k+l-1}, P_{k+l}$ has a total unbalance of $\delta_{k, k+l}=\delta_{k}+\delta_{k+1}+\cdots+\delta_{k+l-1}+\delta_{k+l}$. If $\delta_{k, k+l}>0, \delta_{k, k+l}$ data items must be sent from $C_{k, k+l}$ to the other processors. The ring is unidirectional, so $P_{k+l}$ is the only processor in $C_{k, k+l}$ with an outgoing link. Furthermore, $P_{k+l}$ needs a time equal to $\delta_{k, k+l} \times c$ to send $\delta_{k, k+l}$ data items. Therefore, in any case, a redistribution scheme cannot take less than $\delta_{k, k+l} \times c$ to redistribute all data items. We have the same type of reasoning for the case $\delta_{k, k+l}<0$.

\subsection{An optimal algorithm}

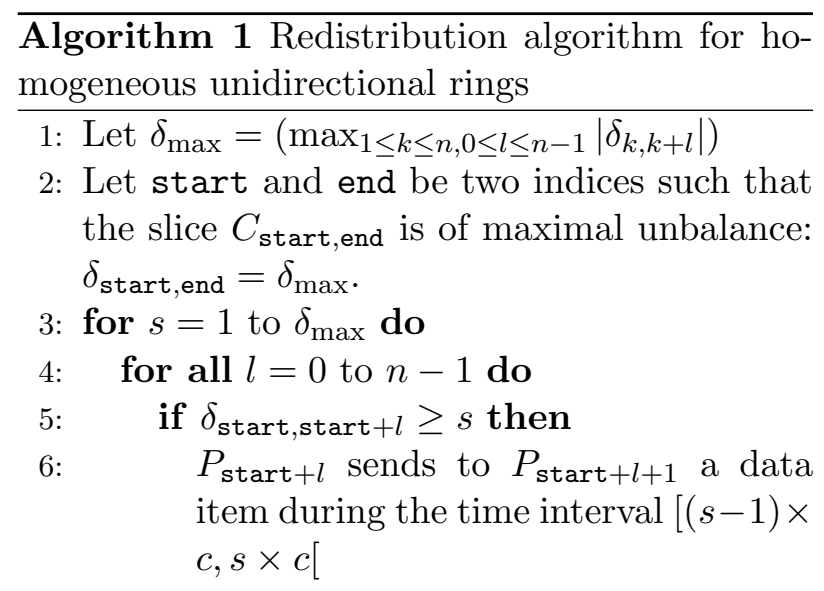

Algorithm 1 is an optimal solution to our problem. We first prove its correctness (Lemma 3). Secondly, we prove its optimality (Lemma 4). Intuitively, if Step 6 of this algorithm is always feasible, then each execution of Step 3 has exactly a length of $c$, and the algorithm will meet the time bound of Lemma 1.

First, we point out that the slice $C_{\text {start,end }}$ is well-defined in Step 2 of the algorithm: for any slice with an unbalance $\delta$, the slice made up from the remaining processors has the opposite unbalance $-\delta$. Next, we state the particular role of the processor $P_{\text {start }}$ :

Lemma 2. Processor $P_{\text {start }}$ receives no data items during the execution of Algorithm 1.

Proof. We prove the result by contradiction. Suppose that at a given iteration $s$ processor $P_{\text {start }}$ receives some data items. Then the predecessor of $P_{\text {start }}$ in the ring, $P_{\text {start }-1}$, sends a data item at this iteration. Thus, $P_{\text {start-1 }}$ being a sender, by the condition at Step 5 of Algorithm 1, $\delta_{\text {start }, \text { start }-1}=\sum_{j=0}^{n-1} \delta_{\text {start }+j} \geq s$. However, 
due to the conservation law, $\sum_{i=1}^{n} \delta_{i}=0$. Hence, $0 \geq s$, the desired contradiction.

To prove that Algorithm 1 is correct, we must show that during each iteration, any processor required to send a data item in Step 6 actually holds at least one data item at this iteration. In other words, we must prove that no processor is asked to send a data item that it does not currently own. Let $L_{i}^{s}$ be the load of $P_{i}$ at the end of iteration $s$ of Algorithm 1:

Lemma 3. During iteration s of loop 3, if $P_{i}$ sends a data item, then $L_{i}^{s-1} \geq 1$.

Proof. We prove Lemma 3 by induction. By definition of unbalances (see Section 2), we know that each processor $P_{i}$ in the ring initially holds an amount of $L_{i}^{0}=L_{i} \geq 1$ data items. Thus the result holds for $s=1$.

Now we suppose that the result holds until a certain iteration $s$ (included), and we focus on iteration $s+1$. There are two cases to consider depending whether processor $P_{i}$ is supposed to receive a data item during iteration $s+1$ or not:

1. If processor $P_{i}$ is both a sender and a receiver during iteration $s+1$, then $P_{i}$ is both a sender and a receiver during iteration $s$ by the condition at Step 5 of Algorithm 1 . Then the load of $P_{i}$ after iteration $s$ was the same than before that iteration and $L_{i}^{s}=L_{i}^{s-1}$. We conclude using the induction hypothesis.

2. If processor $P_{i}$ is a sender but not a receiver during iteration $s+1$, we must verify that $P_{i}$ does not send a data item that it does not hold. Because $P_{i}$ is a sender we have by the condition at Step 5 of Algorithm 1:

$$
\delta_{\text {start }, i} \geq s+1 .
$$

Furthermore, $P_{i}$ has sent a data item during each of the previous iterations.

During iteration $s+1, P_{i}$ is not a receiver. Thus, $P_{i-1}$ is not a sender during this iteration, and, by the condition at Step 5 of Algorithm 1, we have: $\delta_{\text {start }, i-1}<s+1$. During each iteration from 1 to $\delta_{\text {start }, i-1}, P_{i-1}$ has sent a data item (see below for the proof that $\delta_{\text {start,start }+j} \geq 0$ for all $\left.j \in[0, n-1]\right)$. Hence, during each of these iterations, $P_{i}$ was both a sender and a receiver, and neither its load nor its unbalance did change.

During each iteration from $1+\delta_{\text {start }, i-1}$ to $s$, processor $P_{i}$ was a sender but not a receiver. So both its load and its unbalance decrease by one during each of these iterations. Hence:

$$
L_{i}^{s}=L_{i}-\left(s-\delta_{\text {start }, i-1}\right) .
$$

However, $\delta_{i}+\delta_{\text {start }, i-1}=\delta_{\text {start }, i}$. So Equation 3 is equivalent to: $L_{i}^{s}=L_{i}-\delta_{i}+$ $\delta_{\text {start }, i}-s$. From Equation 2 we know that $\delta_{\text {start }, i}-s \geq 1$. In Section 2, we assumed that $L_{i} \geq 1+\delta_{i}$. So, $L_{i}^{s} \geq 2$.

The above proof relies on the property that, for any value of $j \in[0, n-1], \delta_{\text {start,start }+j} \geq$ 0 . We now prove this result by contradiction. Hence we suppose that there exists a value $j$ such that $\delta_{\text {start }, \text { start }+j}<0$. We have two cases to consider:

1. $j+$ start $\in$ [start,end]. Then $\delta_{\text {start,end }}=\delta_{\text {start,start }+j}+\delta_{\text {start }+j+1, \text { end }}$ and $\delta_{\text {start,end }}<\delta_{\text {start }+j+1 \text {,end }}$ which contradicts the maximality of $C_{\text {start,end }}$.

2. $j+$ start $\notin \quad$ start, end]. Then $\delta_{\text {start }, j+\text { start }}=\delta_{\text {start,end }}+\delta_{1+\text { end }, j+\text { start }}$. So $\delta_{\text {start,end }}<-\delta_{1+\text { end, } j+\text { start }}$. However, as the sum of unbalances is null by definition, the sum of unbalances of $C_{1+\text { end, } j+\text { start }}$ is equal to the opposite of the sum of unbalances of $C_{j+1+\text { start,end }}$. Hence, $\delta_{\text {start,end }}<$ $\delta_{j+1+\text { start,end }}$, which contradicts the maximality of $C_{\text {start,end }}$.

We have proved the correction of Algorithm 1. We still have to prove that when it terminates, the entire redistribution has actually been performed: 
Lemma 4. When Algorithm 1 terminates after iteration $\delta_{\max }$, i.e., at time $\tau$, the load of any processor $P_{i}$ is equal to $L_{i}-\delta_{i}$.

Proof. We prove by induction on the processor indices, starting at processor $P_{\text {start }}$, that any processor $P_{j}$ has the desired load of $L_{j}-\delta_{j}$ at any iteration $s \geq \max _{0 \leq i \leq j} \delta_{\text {start,start }+i}$

As stated by Lemma 2, processor $P_{\text {start }}$ never receives a data item during the algorithm execution. So, after $\delta_{\text {start,start }}=\delta_{\text {start }}$ iterations of loop $3, P_{\text {start }}$ is never the receiver nor the sender of a data item. As required, $P_{\text {start }}$ exactly holds $L_{\text {start }}-\delta_{\text {start }}$ data items, i.e., its initial load minus the amount of data items sent.

We suppose the result proved up to a processor $P_{\text {start }+l}$ (with $l \geq 0$ ) included. We focus on processor $P_{\text {start }+l+1}$. Using the induction hypothesis, we know that at any iteration $s \geq$ $\max _{0 \leq i \leq l} \delta_{\text {start,start }+i}$, the total load of the slice $C_{\text {start }, \text { start }+l}$ is equal to $\sum_{0 \leq i \leq l} L_{i}-\sum_{0 \leq i \leq l} \delta_{i}$.

During the execution of the whole algorithm, processor $P_{\text {start }+l+1}$ has sent exactly $\delta_{\text {start,start }+l+1}$ data items (remember that for any $\left.j \in[0, n-1], \delta_{\text {start }, \text { start }+j} \geq 0\right)$. All these send operations took place before or during iteration $\delta_{\text {start,start }+l+1}$. Furthermore, Lemma 2 states that processor $P_{\text {start }}$ never receives a data item during the execution. So, the total load of the slice $C_{\text {start,start }+l+1}$ does not change after iteration $\delta_{\text {start,start }+l+1}$, and its total load is equal to its initial total load minus the data items sent by processor $P_{\text {start }+l+1}$ : $\left(\sum_{0 \leq i \leq l+1} L_{i}\right)-\delta_{\text {start }, \text { start }+l+1}$. Therefore, after any iteration $s$, where $s \geq$ $\max \left(\max _{0 \leq i \leq l} \delta_{\text {start }, \text { start }+i}, \delta_{\text {start }, \text { start }+l+1}\right)=$ $\max _{0 \leq i \leq l+1} \delta_{\text {start,start }+i}$, we know the total load of the slices $C_{\text {start,start }+l}$ and $C_{\text {start,start }+l+1}$. Therefore, we know the load of processor
$P_{\text {start }+l+1}$ at any step $t \geq s:$

$$
\begin{gathered}
L_{\text {start }+l+1}^{t}= \\
\left(\left(\sum_{0 \leq i \leq l+1} L_{\text {start }+i}\right)-\delta_{\text {start }, \text { start }+l+1}\right) \\
\quad-\left(\sum_{0 \leq i \leq l} L_{\text {start }+i}-\sum_{0 \leq i \leq l} \delta_{\text {start }+i}\right) \\
=L_{\text {start }+l+1}-\delta_{\text {start }+l+1 .} .
\end{gathered}
$$

To conclude, we just need to remark that $\delta_{\max }=$ $\max _{0 \leq i \leq n-1} \delta_{\text {start, start }+i}$.

The optimality of Algorithm 1 is a direct consequence of the previous lemmas:

Theorem 1. Algorithm 1 is optimal.

\section{Heterogeneous unidirectional ring}

In this section we still suppose that the ring is unidirectional but we no longer assume the communication paths to have the same capacities. We build on the results of the previous section to design an optimal algorithm (Algorithm 2 below). In this algorithm, the amount of data items sent by any processor $P_{i}$ is exactly the same as in Algorithm 1 (namely $\delta_{\text {start }, i}$ ). However, as the communication links have different capabilities, we no longer have a synchronous behavior. A processor $P_{i}$ sends its $\delta_{\text {start }, i}$ data items as soon as possible, but we cannot express its completion time with a simple formula. Indeed, if $P_{i}$ initially holds more data items than it has to send, we have the same behavior than previously: $P_{i}$ can send its data items during the time interval $\left[0, \delta_{\text {start }, i} \times c_{i, i+1}\right.$ [. On the contrary, if $P_{i}$ holds less data items than it has to send $\left(L_{i}<\delta_{\text {start }, i}\right)$, $P_{i}$ still starts to send some data items at time 0 but may have to wait to have received some other data items from $P_{i-1}$ to be able to forward them to $P_{i+1}$.

The asynchronousness of Algorithm 2 implies that it is correct by construction. Furthermore, 
$\overline{\text { Algorithm } 2 \text { Redistribution algorithm for het- }}$ erogeneous unidirectional rings

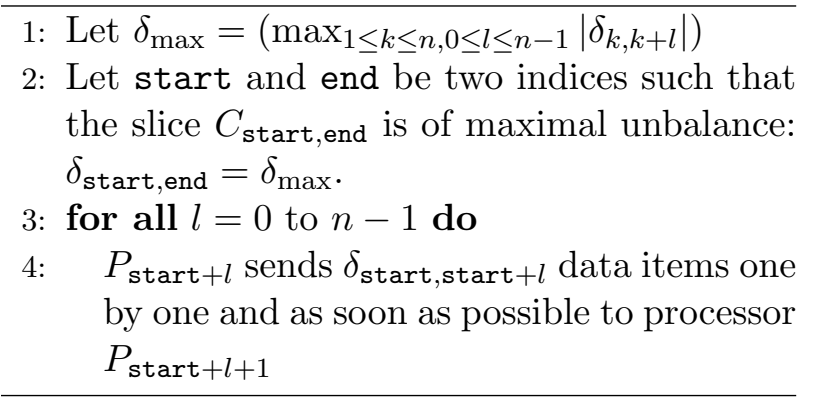

when the algorithm terminates, the redistribution is complete (the proof is the same as in Lemma 4). There remains to prove that the running time of Algorithm 2 is optimal. We first compute this running time:

Lemma 5. The running time of Algorithm 2 is $\max _{0 \leq l \leq n-1} \delta_{\text {start, start }+l} \times c_{\text {start }+l, \text { start }+l+1}$.

The result of Lemma 5 is surprising. Intuitively, it says that the running time of Algorithm 2 is equal to the maximum of the communication times of all the processors, if each of them initially stored locally all the data items it will have to send throughout the execution of the algorithm. In other words, there is no forwarding delay, whatever the initial distribution. The proof of Lemma 5 is technical and can be omitted at first reading.

Proof. We prove the result by contradiction, assuming that the running time of Algorithm 2 , denoted as $t_{\max }$, is strictly greater than $\max _{0 \leq l \leq n-1} \delta_{\text {start,start }+l} \times c_{\text {start }+l, \text { start }+l+1}$ (we assume that the algorithm starts running at time $0)$. Let $P_{i}$ be any processor whose running time is $t_{\max }$, i.e., let $P_{i}$ be any processor which terminates the emission of its last data item at time $t_{\text {max }}$. By hypothesis, $t_{\max }>\delta_{\text {start }, i} \times c_{i, i+1}$. Therefore, there is some time during the running time of the algorithm at which processor $P_{i}$ is not sending any data items to processor $P_{i+1}$. Let $t_{i}$ denote the latest time at which $P_{i}$ is not sending any data items. Then, by definition of $t_{i}$, from time $t_{i}$ until the completion of the algorithm, processor $P_{i}$ is continuously sending data items to $P_{i+1}$. Let $n_{i}$ denote the number of data items that $P_{i}$ sends during that interval. Note that we have $t_{\max }=t_{i}+n_{i} \times c_{i, i+1}$. We now prove by induction that for any value of $j \geq 1$ :

1. Processor $P_{i-j}$ sends a data item to processor $P_{i-j+1}$ during the time interval $\left[t_{i}-\sum_{k=1}^{j} c_{i-k, i-k+1}, t_{i}-\sum_{k=1}^{j-1} c_{i-k, i-k+1}\right]$.

2. Between time $t_{i}-\sum_{k=1}^{j} c_{i-k, i-k+1}$ and the completion of the algorithm, processor $P_{i-j}$ sends at least $j+n_{i}$ data items to processor $P_{i-j+1}$.

3. $c_{i-j, i-j+1} \leq c_{i, i+1}$.

4. Right before time $t_{i}-\sum_{k=1}^{j} c_{i-k, i-k+1}$, processor $P_{i-j}$ is not sending any data items to processor $P_{i-j+1}$ (it is idle in sending).

Once we have proved these properties, the contradiction follows from considering processor $P_{\text {start }}$. Processor $P_{\text {start }}$ only sends data items that it initially holds $\left(\delta_{\text {start }}=\delta_{\text {start,start }} \leq\right.$ $L_{\text {start }}$ ), and receives no data items from its predecessor in the ring. However, using the above properties, there is a value of $j \geq 0$ such that start $=i-j$, and between time $t_{i}-\sum_{k=1}^{j+1} c_{i-k, i-k+1}$ and the completion of the algorithm, processor $P_{i-j-1}$ sends at least $j+1+n_{i}$ data items to processor $P_{i-j}=P_{\text {start }}$. Hence the contradiction.

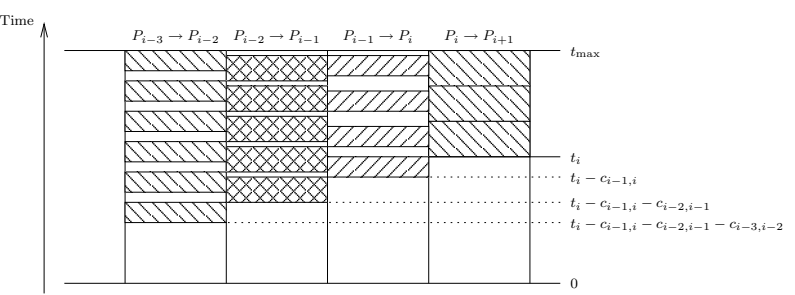

Figure 1: The construction used in the proof of Lemma 5.

The construction used in the proof is illustrated by Figure 1 (where $t_{i-j}=t_{i}-$ 
$\left.\sum_{k=1}^{j} c_{i-k, i-k+1}\right)$. We start by proving the above properties for $j=1$.

1. By definition of $t_{i}$, processor $P_{i}$ is not sending any data items to processor $P_{i+1}$ right before time $t_{i}$. Because of the "as-soon-as" nature of the algorithm, processor $P_{i}$ is not holding a single data item right before time $t_{i}$ and is waiting for processor $P_{i-1}$ to send it one. Furthermore, the data item that processor $P_{i}$ started to send at time $t_{i}$ is sent to it by processor $P_{i-1}$ during the time interval $\left[t_{i}-c_{i-1, i}, t_{i}\right]$.

2. Between time $t_{i}$ and the completion of the algorithm, processor $P_{i}$ sends $n_{i}$ data items to processor $P_{i+1}$. By hypothesis, processor $P_{i}$ holds at least one data item after the completion of the algorithm. As $P_{i}$ holds no data item right before time $t_{i}$, then between the times $t_{i}-c_{i-1, i}$ and $t_{\max }, P_{i-1}$ sends at least $1+n_{i}$ data items to $P_{i}$.

3. From what just precedes, and using the relationship between $t_{i}, n_{i}$, and $t_{\max }$, we have: $t_{i}+n_{i} \times c_{i, i+1}=t_{\max }$ and $t_{\max } \geq$ $\left(t_{i}-c_{i-1, i}\right)+\left(1+n_{i}\right) \times c_{i-1, i}$, which imply $c_{i, i+1} \geq c_{i-1, i}$, as $n_{i}$ is nonzero by definition.

4. Suppose that processor $P_{i-1}$ is sending a data item to processor $P_{i}$ right before the time $t_{i}-c_{i-1, i}$. Then, at the earliest, this data item is received by processor $P_{i}$ at time $t_{i}-c_{i-1, i}$. Due to the "as-soon-as" nature of the algorithm, $P_{i}$ forwards this data item to processor $P_{i+1}$ (as it forwards data items received later). $P_{i}$ finishes to forward this data item at time $t_{i}-c_{i-1, i}+c_{i, i+1} \geq t_{i}$ at the earliest. Therefore, processor $P_{i}$ has no reason not to be sending any data item at time $t_{i}$, which contradicts the definition of $t_{i}$.

We now proceed to the general case of the induction. We suppose that the property is proved up to a processor $P_{i-j}$ included (with $j \geq 1$ ).
1. By induction hypothesis, processor $P_{i-j}$ is not sending any data items to processor $P_{i-j+1}$ right before time $t_{i}-$ $\sum_{k=1}^{j} c_{i-k, i-k+1}$. Because of the "as-soonas" nature of the algorithm, processor $P_{i-j}$ is not holding a single data item right before this time and is waiting for processor $P_{i-j-1}$ to send one. Furthermore, the data item that processor $P_{i-j}$ started to send at time $t_{i}-\sum_{k=1}^{j} c_{i-k, i-k+1}$ is sent to it by processor $P_{i-j-1}$ during the time interval $\left[t_{i}-\sum_{k=1}^{j+1} c_{i-k, i-k+1}, t_{i}-\sum_{k=1}^{j} c_{i-k, i-k+1}\right]$.

2. Between time $t_{i}-\sum_{k=1}^{j} c_{i-k, i-k+1}$ and the completion of the algorithm, processor $P_{i-j}$ sends $j+n_{i}$ data items to processor $P_{i-j+1}$, by induction hypothesis. By hypothesis, processor $P_{i-j}$ holds at least one data item after the completion of the algorithm. As $P_{i-j}$ holds no data item right before time $t_{i}-\sum_{k=1}^{j} c_{i-k, i-k+1}$, then between the times $t_{i}-\sum_{k=1}^{j+1} c_{i-k, i-k+1}$ and $t_{\max }, P_{i-j-1}$ sends at least $1+j+n_{i}$ data items to $P_{i-j}$.

3. From what just precedes, and using the relationship between $t_{i}, n_{i}$, and $t_{\max }$, we have:

$$
\begin{gathered}
t_{i}+n_{i} \times c_{i, i+1}=t_{\max } \geq \\
\left(t_{i}-\sum_{k=1}^{j+1} c_{i-k, i-k+1}\right)+\left(1+j+n_{i}\right) \times c_{i-j-1, i-j}
\end{gathered}
$$

Therefore,

$$
\begin{aligned}
n_{i} \times c_{i, i+1}+\sum_{k=1}^{j} c_{i-k, i-k+1} & \geq \\
\left(j+n_{i}\right) & \times c_{i-j-1, i-j}
\end{aligned}
$$

and thus: $c_{i, i+1} \geq c_{i-j-1, i-j}$ as, by induction hypothesis, for any $k \in[1, j], c_{i, i+1} \geq$ $c_{i-k, i-k+1}$.

4. Suppose that processor $P_{i-j-1}$ is sending a data item to processor $P_{i-j}$ right before the time $t_{i}-\sum_{k=1}^{j+1} c_{i-k, i-k+1}$. Then, at the earliest, this data item is received by processor 
$P_{i-j}$ at time $t_{i}-\sum_{k=1}^{j+1} c_{i-k, i-k+1}$. Due to the "as-soon-as" nature of the algorithm, $P_{i-j}$ forwards this data item to processor $P_{i-j+1}$ (as it forwards data items received later). $P_{i-j}$ finishes to forward this data item at time $t_{i}-c_{i-j-1, i-j}-\sum_{k=1}^{j-1} c_{i-k, i-k+1}$ at the earliest. Then, following the same line of reasoning, processor $P_{i-j+1}$ forwards it to $P_{i-j+2}$, which receives it at the earliest at time $t_{i}-c_{i-j-1, i-j}-\sum_{k=1}^{j-2} c_{i-k, i-k+1}$, and so on. So, processor $P_{i}$ receives this data item at the earliest at time $t_{i}-c_{i-j-1, i-j}$, and forwards it. Then, it finishes to send it at the earliest at time $t_{i}-c_{i-j-1, i-j}+c_{i, i+1} \geq t_{i}$, as we have seen that $c_{i, i+1} \geq c_{i-j-1, i-j}$. Therefore, processor $P_{i}$ has no reason not to be sending any data items at time $t_{i}$, which contradicts the definition of $t_{i}$. Hence, processor $P_{i-j-1}$ is not sending any data item to processor $P_{i-j}$ right before the time $t_{i}-$ $\sum_{k=1}^{j+1} c_{i-k, i-k+1}$.

Theorem 2. Algorithm 2 is optimal.

Proof. Let $\tau$ denote the optimal redistribution time. Following the arguments used in the proof of Lemma 1 for the homogeneous case in Section 3.1, we obtain the lower bound:

$$
\tau \geq \max _{1 \leq k \leq n, 0 \leq l \leq n-1}\left|\delta_{k, k+l}\right| \times c_{k+l, k+l+1} .
$$

We conclude using Lemma 5.

\section{Homogeneous bidirectional ring}

In this section, we consider a homogeneous bidirectional ring. All links have the same capacity but a processor can send data items to its two neighbors in the ring: there exists a constant $c$ such that, for all $i \in[1, n], c_{i, i+1}=c_{i, i-1}=c$. We proceed as for the homogeneous unidirectional case: we first derive a lower bound on the running time of any redistribution algorithm, and then we present an algorithm attaining this bound.

\subsection{Lower bound}

We have the following bound on the optimal redistribution time:

Lemma 6. Let $\tau$ be the optimal redistribution time. Then:

$$
\tau \geq \max \left\{\max _{1 \leq i \leq n}\left|\delta_{i}\right|, \max _{\substack{1 \leq i \leq n, 1 \\ 1 \leq l \leq n-1}}\left\lceil\frac{\left|\delta_{i, i+l}\right|}{2} \mid\right\} \times c .\right.
$$

Proof. Consider any processor $P_{i}$ with positive unbalance $\left(\delta_{i}>0\right)$. Even if processor $P_{i}$ can send data items to both of its neighbors, because of the one-port model, it cannot send data items to both of them simultaneously. So, it requires processor $P_{i}$ at least a time of $\delta_{i} \times c$ to send $\delta_{i}$ data items, whatever the destinations of these data items. We have a symmetric result for the case $\delta_{i}<0$. Hence a first lower-bound on the optimal redistribution time $\tau$ :

$$
\tau \geq\left(\max _{1 \leq i \leq n}\left|\delta_{i}\right|\right) \times c .
$$

Now, consider any non trivial slice of consecutive processors $C_{k, l}$. By "non trivial" we mean that the slice is not reduced to a single processor (we already considered that case) and that it does not contain all processors. We suppose that $\delta_{k, l}>0$. So, in any redistribution scheme, at least $\delta_{k, l}$ data items must be sent by $C_{k, l}$. As this slice is not reduced to a single processor, the two processors at the extremities of the slice, $P_{k}$ and $P_{l}$, can simultaneously send data items to their neighbors outside of the slice, $P_{k-1}$ and $P_{l+1}$ respectively. Therefore, during any time interval of length $c$, at most two data items can be sent from the slice. So, it takes at least a time of $\left\lceil\frac{\delta_{k, l}}{2}\right\rceil \times c$ for the slice $C_{k, l}$ to send $\delta_{k, l}$ data items. Once again, the reasoning is similar when receiving data items if $\delta_{k, l}<0$. Hence a second 
lower-bound on $\tau$ :

$$
\tau \geq\left(\max _{1 \leq i \leq n, 1 \leq l \leq n-1}\left\lceil\frac{\left|\delta_{i, i+l}\right|}{2}\right\rceil\right) \times c .
$$

We just gather the previous two lower-bounds to obtain the desired bound.

\subsection{An optimal algorithm}

Algorithm 3 is a recursive algorithm which defines communication patterns designed so as to decrease the value of $\delta_{\max }$ (computed at Step 1) by one from one recursive call to another. The intuition behind Algorithm 3 is the following:

1. Any non trivial slice $C_{k, l}$ such that $\left\lceil\frac{\left|\delta_{k, l}\right|}{2}\right\rceil=$ $\delta_{\max }$ and $\delta_{k, l} \geq 0$ must send two data items per recursive call, one through each of its extremities.

2. Any non trivial slice $C_{k, l}$ such that $\left\lceil\frac{\left|\delta_{k, l}\right|}{2}\right\rceil=$ $\delta_{\max }$ and $\delta_{k, l} \leq 0$ must receive two data items per recursive call, one through each of its extremities.

3. Once the mandatory communications specified by the two previous cases are defined, we take care of any processor $P_{i}$ such that $\left|\delta_{i}\right|=\delta_{\max }$. If $P_{i}$ is already involved in a communication due to the previous cases, everything is settled. Otherwise, we have the freedom to choose whom $P_{i}$ will send a data item to (case $\delta_{i}>0$ ) or whom $P_{i}$ will receive a data item from $\left(\right.$ case $\left.\delta_{i}<0\right)$. To simplify the algorithm we decide that all these communications will take place in the direction from $P_{i}$ to $P_{i+1}$.

Algorithm 3 is initially called with the parameter $s=1$. For any call to Algorithm 3, all the communications take place in parallel and exactly at the same time, because the communication paths are homogeneous by hypothesis. One very important point about Algorithm 3 is that this algorithm is a set of rules which only specify which processor $P_{i}$ must send a data item to which processor $P_{j}$, one of its immediate neighbors. Therefore, whatever the number of rules deciding that there must be some data item sent from a processor $P_{i}$ to one of its immediate neighbor $P_{j}$, only one data item is sent from $P_{i}$ to $P_{j}$ to satisfy all these rules.

To prove that Algorithm 3 is optimal, we show that the set of rules is consistent, i.e., that it respects the one-port model, and that the value $\delta_{\max }$ (computed at Step 1) decreases by one at each recursive call:

Lemma 7. Algorithm 3 satisfies to all the oneport constraints.

Lemma 8. Algorithm 3 terminates in exactly $\max \left\{\max _{1 \leq i \leq n}\left|\delta_{i}\right|, \max _{1 \leq i \leq n, 1 \leq l \leq n-1}\left\lceil\frac{\delta_{i, i+l}}{2}\right\rceil\right\}$ recursive calls.

The optimality of Algorithm 3 is then a simple corollary of Lemma 8 and of the lower bound defined by Equation 5 (the missing proofs can be found in [30]).

Theorem 3. Algorithm 3 is optimal.

\section{Heterogeneous bidirectional ring}

In this section, we consider the most general case, that of a heterogeneous bidirectional ring. We do not know any optimal redistribution algorithm in this case. However, if we assume that each processor initially holds more data than it needs to send during the whole execution of the algorithm (what we call a light redistribution), then we succeed in deriving an optimal solution.

\subsection{Light redistribution}

Throughout this section, we suppose that we have a light redistribution: we assume that the number of data items sent by any processor throughout the redistribution algorithm is less than or equal to its original load. There are two reasons for a processor $P_{i}$ to send data: (i) because it is overloaded $\left(\delta_{i}>0\right)$; (ii) because it 
has to forward some data to another processor located further in the ring. If $P_{i}$ initially holds at least as many data items as it will send during the whole execution, then $P_{i}$ can send at once all these data items. Otherwise, in the general case, some processors may wait to have received data items from a neighbor before being able to forward them to another neighbor.

\subsubsection{Solution by integer linear program- ming}

Under the "light redistribution" assumption, we can build an integer linear program to solve our problem (see System 6). Let $\mathcal{S}$ be one of its solutions, and denote by $\mathcal{S}_{i, i+1}$ the number of data items that processor $P_{i}$ sends to processor $P_{i+1}$. Similarly, $\mathcal{S}_{i, i-1}$ is the number of data items that $P_{i}$ sends to processor $P_{i-1}$. In order to ease the writing of the equations, we impose in the first two equations of System 6 that $\mathcal{S}_{i, i+1}$ and $\mathcal{S}_{i, i-1}$ are nonnegative for all $i$, which imposes to use other variables $\mathcal{S}_{i+1, i}$ and $\mathcal{S}_{i-1, i}$ for the symmetric communications. The third equation states that after the redistribution, there is no more unbalance. We denote by $\tau$ the execution time of the redistribution. For any processor $P_{i}$, due to the one-port constraints, $\tau$ must be greater than the time spent by $P_{i}$ to send data items (fourth equation) or spent by $P_{i}$ to receive data items (fifth equation). Our aim is to minimize $\tau$, hence the system:

$$
\begin{gathered}
\text { Minimize } \tau, \text { SUBJECT TO } \\
\left\{\begin{array}{l}
\forall i, \mathcal{S}_{i, i+1} \geq 0 \\
\forall i, \mathcal{S}_{i, i-1} \geq 0 \\
\forall i, \mathcal{S}_{i, i+1}+\mathcal{S}_{i, i-1}-\mathcal{S}_{i+1, i}-\mathcal{S}_{i-1, i}=\delta_{i} \\
\forall i, \mathcal{S}_{i, i+1} c_{i, i+1}+\mathcal{S}_{i, i-1} c_{i, i-1} \leq \tau \\
\forall i, \mathcal{S}_{i+1, i} c_{i+1, i}+\mathcal{S}_{i-1, i} c_{i-1, i} \leq \tau
\end{array}\right.
\end{gathered}
$$

Lemma 9. Any optimal solution of System 6 is feasible, for example using the following schedule: for any $i \in[1, n], P_{i}$ starts sending data items to $P_{i+1}$ at time 0 and, after the completion of this communication, starts sending data items to $P_{i-1}$ as soon as possible under the oneport model.
Proof. We have to show that we are able to schedule the communications defined by any optimal solution $(\mathcal{S}, \tau)$ of System 6 so that the redistribution takes a time no greater than $\tau$. For any $i \in[1, n]$, we schedule at time 0 all emissions from $P_{i}$ to $P_{i+1}$. This communication is done in time $\mathcal{S}_{i, i+1} c_{i, i+1}$ : because of the "light redistribution" hypothesis, $P_{i}$ already holds all the data items that it must send. Because of the fourth equation of System 6, this communication ends before the time $\tau$.

For any value of $i \in[1, n]$, we still have to schedule the sending of data items from $P_{i}$ to $P_{i-1}$. We schedule this communication as soon as possible, therefore at time $\max \left\{\mathcal{S}_{i, i+1} c_{i, i+1}\right.$, $\left.\mathcal{S}_{i-2, i-1} c_{i-2, i-1}\right\}$, i.e., at the earliest time when (i) $P_{i}$ has ended sending data items to $P_{i+1}$, and (ii) $P_{i-1}$ has stopped receiving data items from $P_{i-2}$. Therefore, the communication from $P_{i}$ to $P_{i-1}$ ends at the date:

$$
\begin{gathered}
\max \left\{\mathcal{S}_{i, i+1} c_{i, i+1}, \mathcal{S}_{i-2, i-1} c_{i-2, i-1}\right\}+\mathcal{S}_{i, i-1} c_{i, i-1} \\
=\max \left\{\mathcal{S}_{i, i+1} c_{i, i+1}+\mathcal{S}_{i, i-1} c_{i, i-1},\right. \\
\left.\mathcal{S}_{i-2, i-1} c_{i-2, i-1}+\mathcal{S}_{i, i-1} c_{i, i-1}\right\} .
\end{gathered}
$$

Once again, this is true owing to the "light redistribution" hypothesis: no processor needs to wait to have received some data items before being able to send them to one of its neighbors.

The first term of the "max" expression is the time needed by $P_{i}$ to send data items to both $P_{i+1}$ and $P_{i-1}$. This term is less than or equal to $\tau$ because of the fourth equation of System 6 . The second term of the "max" expression is the time needed by $P_{i-1}$ to receive data items from both $P_{i-2}$ and $P_{i}$. This term is less than or equal to $\tau$ because of the fifth equation of System 6 .

So far, we did not mathematically define a condition for the "light redistribution" hypothesis to hold. In fact, this is not mandatory: we use System 6 to find an optimal solution to the problem. If, in this optimal solution, for any processor $P_{i}$, the total number of data items sent is less than or equal to the initial load $\left(\mathcal{S}_{i, i+1}+\mathcal{S}_{i, i-1} \leq L_{i}\right)$, 
we are under the "light redistribution" hypothesis and we can use the solution of System 6 safely.

\subsubsection{Solution through rational linear programming}

Even if the "light redistribution" hypothesis holds, one may wish to solve the redistribution problem with a technique less expensive than integer linear programming (which is potentially exponential). An idea would be to first solve System 6 to find an optimal rational solution, which can always be done in polynomial time, and then to round up the obtained solution to find a "good" integer solution. In fact, the following theorem shows that one of the two natural ways of rounding always lead to an optimal (integer) solution. The complexity of the light redistribution problem is therefore polynomial.

Theorem 4. Let $\mathcal{R}$ be an optimal rational solution to the redistribution problem. For any $j$ in $[1, n], \mathcal{R}_{j}$ denotes the number of data items that processor $P_{j}$ sends to processor $P_{j+1}$ (using the notations of System $\left.6, \mathcal{R}_{j}=\mathcal{S}_{j, j+1}-\mathcal{S}_{j+1, j}\right)$. Let $\mathcal{F}$ be the integer solution defined by $\mathcal{F}_{1}=$ $\left\lfloor\mathcal{R}_{1}\right\rfloor$. Let $\mathcal{G}$ be the integer solution defined by $\mathcal{G}_{1}=\left\lceil\mathcal{R}_{1}\right\rceil$. Then:

(i) $\mathcal{F}$ and $\mathcal{G}$ are well-defined by the single condition above,

(ii) either $\mathcal{F}$ or $\mathcal{G}$ is an optimal integer solution.

Proof. Lemma 10 below states that $\mathcal{F}$ and $\mathcal{G}$ are both fully defined. Lemma 11 below states that there exists at least one optimal integer solution $\mathcal{E}$ such that $\left|\mathcal{E}_{1}-\mathcal{R}_{1}\right|<1$. The only two solutions satisfying these constraints are $\mathcal{F}$ and $\mathcal{G}$. Hence the result.

Lemma 10. To fully define the number of data items sent between processors in any redistribution scheme, we only need to define, for a single given value of $j \in[1, n]$, the number of data items that processor $P_{j}$ sends to processor $P_{j+1}$.

Lemma 11. Let $\mathcal{R}$ be an optimal rational solution to the redistribution problem: for any $j$ in
$[1, n], \mathcal{R}_{j}$ denotes the number of data items processor $P_{j}$ sends to processor $P_{j+1}$. Then, there exists an optimal integer solution $\mathcal{E}$ to the solution problem such that: $\left|\mathcal{E}_{1}-\mathcal{R}_{1}\right|<1$.

The missing proofs can be found in [30].

\subsection{General case}

\subsubsection{Lower bound}

We have the following bound on the optimal redistribution time:

Lemma 12. Let $\tau$ be the optimal redistribution time. Then: $\tau \geq \max _{1 \leq k \leq n, \delta_{k}>0} \delta_{k}$. $\min \left\{c_{k, k-1}, c_{k, k+1}\right\}$

$$
\begin{aligned}
& \tau \geq \max _{1 \leq k \leq n, \delta_{k}<0}-\delta_{k} \cdot \min \left\{c_{k-1, k}, c_{k+1, k}\right\} \\
& \tau \geq \max _{\substack{1 \leq k \leq n, 0 \\
1<l<n-2,}} \min _{0 \leq i \leq \delta_{k, k+l}} \\
& \delta_{k}, k+l>0 \\
& \max \left\{i \cdot c_{k, k-1},\left(\delta_{k, k+l}-i\right) \cdot c_{k+l, k+l+1}\right\} \\
& \tau \geq \max _{1 \leq k \leq n, \quad} \min _{0 \leq i \leq-\delta_{k, k+l}} \\
& \begin{array}{c}
1 \leq \bar{l} \leq \bar{n}-2, \\
\delta_{k}, k+l
\end{array} \\
& \max \left\{i \cdot c_{k-1, k},\left(-\delta_{k, k+l}-i\right) \cdot c_{k+l+1, k+l}\right\}
\end{aligned}
$$

Proof. Consider any processor $P_{i}$ with positive unbalance $\left(\delta_{i}>0\right)$. Even if processor $P_{i}$ can send data items to both of its neighbors, because of the one-port model, it cannot send data items to both of them simultaneously. The best way for processor $P_{i}$ to send $\delta_{i}$ data items is then to send them using the fastest of its outgoing links. So, it requires processor $P_{i}$ at least a time of $\delta_{i} \times \min \left\{c_{i, i-1}, c_{i, i+1}\right\}$ to send $\delta_{i}$ data items, whatever the destinations of these data items. We have a symmetric result for the case $\delta_{i}<0$. Hence the first two inequations on $\tau$.

Now, consider any non trivial slice of consecutive processors $C_{k, l}$. By "non trivial" we mean that the slice is not reduced to a single processor (we already considered that case) and that it 
does not contain all processors. We suppose that $\delta_{k, l}>0$. So, in any redistribution scheme, at least $\delta_{k, l}$ data items must be sent by $C_{k, l}$. As this slice is not reduced to a single processor, the two processors at the extremities of the slice, $P_{k}$ and $P_{l}$, can simultaneously send data items to their neighbors outside of the slice, $P_{k-1}$ and $P_{l+1}$ respectively. Therefore, during the redistribution, processor $P_{k}$ sends a certain amount $i \in\left[0, \delta_{k, l}\right]$ of data items to processor $P_{k-1}$, while processor $P_{l}$ sends the remaining data items to $P_{l+1}$, which takes a time $\max \left\{i \cdot c_{k, k-1},\left(\delta_{k, l}-i\right) \cdot c_{l, l+1}\right\}$. Then we chose for $i$ a value which minimizes this time. We have a symmetric result for the case $\delta_{k, l}<0$. Hence the last two inequations on $\tau$.

\subsubsection{Heuristic approaches}

We do not know whether the bound given by Lemma 12 can always be reached, but we have no counter-example proving that the bound is not tight.

When the solution found by System 6 does not satisfy the "light redistribution" hypothesis, there is the possibility to modify the system to enforce it: we obtain System 8 which finds a solution which satisfies the "light redistribution" hypothesis, if one exists. But there is no reason $a$ priori for the solution of System 8 to be optimal.

$$
\begin{gathered}
\text { Minimize } \tau, \text { SUBJECT TO } \\
\left\{\begin{array}{l}
\forall i, \mathcal{S}_{i, i+1} \geq 0 \\
\forall i, \mathcal{S}_{i, i-1} \geq 0 \\
\forall i, \mathcal{S}_{i, i+1}+\mathcal{S}_{i, i-1}-\mathcal{S}_{i+1, i}-\mathcal{S}_{i-1, i}=\delta_{i} \\
\forall i, \mathcal{S}_{i, i+1} c_{i, i+1}+\mathcal{S}_{i, i-1} c_{i, i-1} \leq \tau \\
\forall i, \mathcal{S}_{i+1, i} c_{i+1, i}+\mathcal{S}_{i-1, i} c_{i-1, i} \leq \tau \\
\forall i, \mathcal{S}_{i, i+1}+\mathcal{S}_{i, i-1} \leq L_{i}
\end{array}\right.
\end{gathered}
$$

To conclude this section, we point out that the design of an optimal algorithm in the most general case remains open. Given the complexity of the lower bound, the problem looks very difficult to solve.

\section{Related work}

Redistribution algorithms have been the focus of an abundant literature. On the theoretical side, in the framework of High Performance Fortran [21] compilation, Kremer [22] showed the NP-completeness of a simple redistribution problem. This negative results shows that optimal algorithms can be designed only for particular cases, such as the ring architecture in this paper. To the best of our knowledge, no other redistribution algorithms has been proven optimal, but several efficient algorithms have been designed for rings $[15,24,10]$, trees or hypercubes [38]. The elastic load balancing algorithm designed in $[25,4]$ has led to a data redistribution software used for query processing [7] and medical image analysis [32].

The block-cyclic distribution of data arrays plays a very important role in scientific libraries [5]. In a CYCLIC $(r)$ distribution over $p$ processors, blocks of $r$ consecutive elements of the array are distributed to the processors in a wraparound fashion, and the parameter $r$ is chosen to optimize the granularity, i.e., the computation-to-communication ratio. Because this granularity changes from one computational kernel to the other, moving from a CYCLIC ( $r$ ) distribution over $p$ processors to a CYCLIC ( $\mathrm{s}$ ) distribution over $q$ processors is a very useful redistribution procedure, which has been implemented using a caterpillar algorithm in ScaLAPACK [29]. Several papers, including $[19,36,11,28,14,16,20]$, have dealt with various optimizations of this redistribution procedure. Along this line of research, automatic data redistribution tools are presented in [14].

Even though we did not deal with loadbalancing algorithms in this paper, we quote some key references on the subject. For homogeneous platforms, see the collection of papers [35], and for heterogeneous clusters see chapter 25 in [8]. Several authors $[12,27,26,37,17]$ propose a mapping policy which dynamically minimizes system degradation (including the cost of remapping) for each computation step. Static strate- 
gies aiming at distributing independent chunks of work to two-dimensional processor grids are studied in $[1,2]$. Relaxing the geometrical constraints induced by two-dimensional grids leads to irregular partitionings [9, 18, 3] that allow for a good load-balancing but are much more difficult to implement. This approach has been extended to three-dimensional problems [13].

Finally, we briefly mention three sample applications whose implementation can directly benefit from the redistribution strategies designed in this paper. The analysis of pulses propagating in a nonlinear medium calls for adaptive computational windows, and redistribution must occur frequently as the computation progresses [6]. A two-level redistribution procedure is advocated in [23] for structured adaptive mesh refinement. A multi-level diffusion re-partitioner is presented in $[33,34]$ for irregular grid computations and has been incorporated into the PARMETIS library. Of course this short list could be extended dramatically.

\section{Simulation results}

Due to lack of space, we refer the reader to $[31,30]$ for the details. As expected, when the computation to communication ratio is high, the best strategy is to use no redistribution, as their cost is prohibitive. Conversely, when the computation to communication ratio is low, it pays off to use many redistributions, but not too many! As the ratio decreases, all tradeoffs can be found.

\section{Conclusion}

We have considered the problem of redistributing data on rings of processors. For homogeneous rings the problem has been completely solved. Indeed, we have designed optimal algorithms, and provided formal proofs of correctness, both for unidirectional and bidirectional rings. The bidirectional algorithm turned out to be quite complex, and requires a lengthy proof.
For heterogeneous rings there remains further research to be conducted. The unidirectional case was easily solved, but the bidirectional case remains open. Still, we have derived an optimal solution for light redistributions, an important case in practice. The complexity of the bound for the general case shows that designing an optimal algorithm is likely to be a difficult task.

All our algorithms have been implemented and extensively tested. As expected, the cost of data redistributions may not pay off a little unbalance of the work in some cases. Further work will aim at investigating how frequently redistributions must occur in real-life applications.

\section{References}

[1] J. Barbosa, J. Tavares, and A. J. Padilha. Linear algebra algorithms in a heterogeneous cluster of personal computers. In HCW'2000, pages 147-159. IEEE CS Press, 2000 .

[2] O. Beaumont, V. Boudet, A. Petitet, F. Rastello, and Y. Robert. A proposal for a heterogeneous cluster ScaLAPACK (dense linear solvers). IEEE Trans. Computers, 50(10):1052-1070, 2001.

[3] O. Beaumont, V. Boudet, F. Rastello, and Y. Robert. Matrix multiplication on heterogeneous platforms. IEEE TPDS, 12(10):1033-1051, 2001.

[4] A. Bevilacqua. A dynamic load balancing method on a heterogeneous cluster of workstations. Informatica, 23(1):49-56, 1999.

[5] L. S. Blackford, J. Choi, A. Cleary, E. D'Azevedo, J. Demmel, I. Dhillon, J. Dongarra, S. Hammarling, G. Henry, A. Petitet, K. Stanley, D. Walker, and R. C. Whaley. ScaLAPACK Users' Guide. SIAM, 1997.

[6] A. Bourgeade and B. Nkonga. Dynamic load balancing computation of pulses propagat- 
ing in a nonlinear medium. The Journal of Supercomputing, 28(3):279-294, 2004.

[7] L. Brunie, A. Flory, and H. Kosch. New static scheduling and elastic load balancing methods for parallel query processing. In BIWIT. IEEE CS Press, 1995.

[8] R. Buyya. High Performance Cluster Computing. Volume 1: Architecture and Systems. Prentice Hall PTR, Upper Saddle River, NJ, 1999.

[9] P. E. Crandall and M. J. Quinn. Block data decomposition for data-parallel programming on a heterogeneous workstation network. In $H P D C$, pages 42-49. IEEE CS Press, 1993.

[10] E. Deelman and B. Szymanski. Dynamic load balancing in parallel discrete event simulation for spatially explicit problems. In PADS'98, pages 46-53. IEEE CS Press, 1998.

[11] F. Desprez, J. Dongarra, A. Petitet, C. Randriamaro, and Y. Robert. Scheduling blockcyclic array redistribution. IEEE TPDS, 9(2):192-205, 1998.

[12] J. E. Flaherty, R. M. Loy, C. Özturan, M. S. Shephard, B. K. Szymanski, J. D. Teresco, and L. H. Ziantz. Parallel structures and dynamic load balancing for adaptive finite element computation. Applied Numerical Mathematics, 26(1-2):241-263, 1997.

[13] J. E. Flaherty, R. M. Loy, M. S. Shephard, B. K. Szymanski, J. D. Teresco, and L. H. Ziantz. Adaptive local refinement with octree load balancing for the parallel solution of three-dimensional conservation laws. J. Parallel and Distributed Computing, 47(2):139-152, 1997.

[14] J. Garcia, E. Ayguadé, and J. Labarta. A framework for integrating data alignment, distribution, and redistribution in distributed memory multiprocessors. IEEE TPDS, 12(4):416-431, 2001.

[15] M. Hamdi and C. Lee. Dynamic load balancing of data parallel applications on a distributed network. In ICS'95, pages 170-179. ACM Press, 1995.

[16] C. Hsu, Y. Chung, D. Yang, and C. Dow. A generalized processor mapping technique for array redistribution. IEEE TPDS, 12(7):743-757, 2001.

[17] Y. Hu and R. Blake. Load balancing for unstructured mesh applications. Parallel and Distributed Computing Practices, 2(3), 1999.

[18] M. Kaddoura, S. Ranka, and A. Wang. Array decomposition for nonuniform computational environments. Journal of Parallel and Distributed Computing, 36:91-105, 1996.

[19] E. T. Kalns and L. M. Ni. Processor mapping techniques towards efficient data redistribution. IEEE TPDS, 6(12):1234-1247, 1995.

[20] J. Knoop and E. Mehofer. Distribution assignment placement: effective optimization of redistribution costs. IEEE TPDS, 13(6):628-647, 2002.

[21] C. H. Koelbel, D. B. Loveman, R. S. Schreiber, G. L. S. Jr., and M. E. Zosel. The High Performance Fortran Handbook. The MIT Press, 1994.

[22] U. Kremer. NP-Completeness of dynamic remapping. In Proceedings of the Fourth Workshop on Compilers for Parallel Computers, Delft, The Netherlands, 1993. Also available as Rice Technical Report CRPCTR93330-S.

[23] Z. Lan, V. Taylor, and G. Bryan. Dynamic load balancing of samr applications on distributed systems. In Proceedings of $S C^{\prime} 01$. IEEE CS Press, 2001. 
[24] C. Lee and M. Hamdi. Parallel image processing applications on a network of workstations. Parallel Computing, 21:137-160, 1995.

[25] S. Miguet and Y. Robert. Elastic load balancing for image processing algorithms. In H. Zima, editor, Parallel Computation, LNCS 591, pages 438-451. Springer Verlag, 1992.

[26] D. Nicol and J. P.F. Reynolds. Optimal dynamic remapping of data parallel computations. IEEE Trans. Computers, 39(2):206219, 1990.

[27] D. Nicol and J. Saltz. Dynamic remapping of parallel computations with varying resource demands. IEEE Trans. Computers, 37(9):1073-1087, 1988.

[28] N. Park, V. Prasanna, and C. Raghavendra. A framework for integrating data alignment, distribution, and redistribution in distributed memory multiprocessors. IEEE TPDS, 10(12):1217-1240, 1999.

[29] L. Prylli and B. Tourancheau. Fast runtime block-cyclic data redistribution on multiprocessors. J. Parallel Distributed Computing, 45:63-72, 1997.

[30] H. Renard, Y. Robert, and F. Vivien. Data redistribution algorithms for homogeneous and heterogeneous processor rings. Research Report 5207, INRIA, May 2004.

[31] H. Renard, Y. Robert, and F. Vivien. Data redistribution algorithms for homogeneous and heterogeneous processor rings. In HiPC'2004, volume 3296 of $L N C S$, pages 123-132. Springer Verlag, 2004.

[32] D. Sarrut and S. Miguet. ARAMIS: a remote access medical imaging system. In $I S$ COPE'99, volume 1732 of LNCS. Springer, 1999.
[33] K. Schloegel, G. Karypis, and V. Kumar. Multilevel diffusion schemes for repartitioning of adaptive meshes. Journal of Parallel and Distributed Computing, 47:109-124, 1997.

[34] K. Schloegel, G. Karypis, and V. Kumar. A unified algorithm for load-balancing adaptive scientific simulations. In Proceedings of $S C$ '00. IEEE CS Press, 2000.

[35] B. A. Shirazi, A. R. Hurson, and K. M. Kavi. Scheduling and load balancing in parallel and distributed systems. IEEE CS Press, 1995.

[36] R. Thakur, A. Choudhary, and J. Ramanujam. Efficient algorithms for array redistribution. IEEE TPDS, 7(6):587-594, 1996.

[37] J. Watts and S. Taylor. A practical approach to dynamic load balancing. IEEE TPDS, 9(93):235-248, 1998.

[38] M.-Y. Wu. On runtime parallel scheduling for processor load balancing. IEEE TPDS, 8(2):173-186, 1997. 


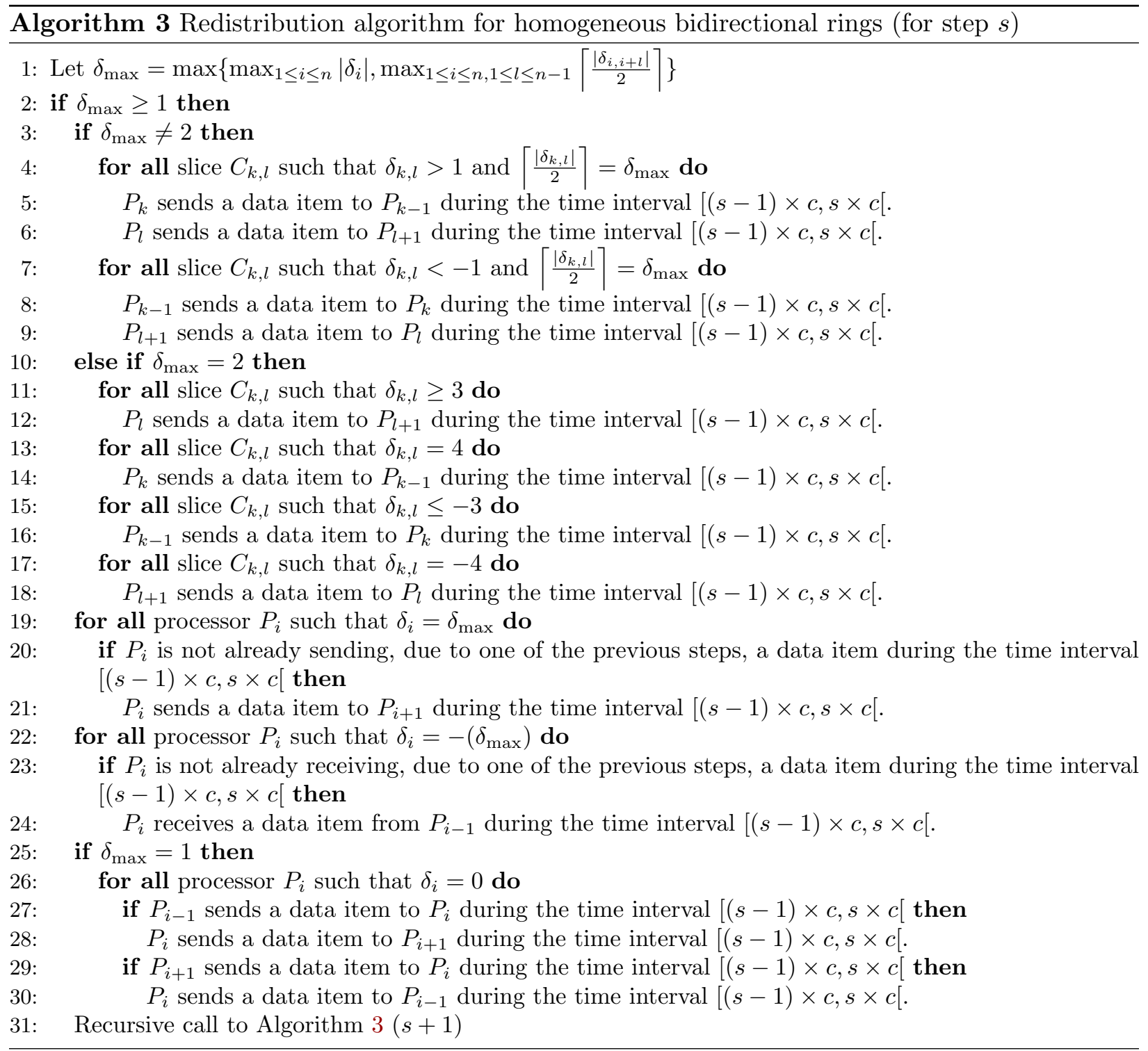

